\title{
¿Qué es la innovación social? El cambio de paradigma y su relación con el Trabajo Social
}

\author{
What is social innovation? The paradigm shift and its relationship \\ with Social Work \\ Andoni Alonso Puelles \\ Universidad Complutense de Madrid, España \\ andonial@ucm.es \\ Javier ECHEVERRÍA EZPONDA \\ Universidad del País Vasco, España \\ jecheverria@sinnergiak.org
}

Recibido: $28 / 01 / 2016$

Revisado: 17/02/206

Aceptado: 13/05/2016

Disponible on line: 01/07/2016

\section{Resumen}

La innovación forma parte de la esencia del Trabajo Social. En los últimos años el paradigma de innovación ha cambiado, por lo que resulta más fácil de aceptar esta relación. Las políticas estatales y supraestatales de fomento de la innovación parecen dirigirse precisamente en ese sentido. Para poder afirmar tal hipótesis es necesario replantear la percepción que los y las trabajadoras sociales tienen de su tarea. También es útil encontrar casos particulares que muestren cómo se producen tales innovaciones sociales.

Palabras clave: innovación social, cambio de paradigma, políticas, Trabajo Social.

\begin{abstract}
Innovation is a fundamental part of social work. In recent years there has been a shift in the innovation paradigm, making it easier to accept this relationship. National and supranational policies aimed at promoting innovation appear to be specifically guided by this idea. To be able to affirm this hypothesis, it is necessary to review the perception that social workers have of their duties. It is also useful to examine particular cases that show how such social innovation arises.
\end{abstract}

Keywords: social innovation, paradigm shift, policies, Social Work.

Referencia normalizada: Alonso Puelles, A. y Echeverría Ezponda, J. (2016): «¿Qué es la innovación social? El cambio de paradigma y su relación con el Trabajo Social». Cuadernos de Trabajo Social, 29(2): 163-171.

Sumario: Introducción. 1. La ampliación del campo en los estudios de innovación. 2. La irrupción de la innovación social. 3. Excurso sobre el Trabajo Social y la innovación tecnológica de las tecnologías de la información y la comunicación (TIC). 4. La innovación y el Trabajo Social. 5. Caso de estudio. 6. Conclusiones. 7. Referencias bibliográficas.

\section{Introducción}

Este monográfico centra su atención en la relación, si existe entre la innovación, la tecnología y el Trabajo Social. Ciertamente un estudio profundo de esta cuestión no es posible en este número, dada la extensión del tema. En la actualidad la bibliografía que aborda la innovación resulta inabarcable, por lo que parece sensato tratar de acotar el campo de investigación. Está de moda hablar de innovación e incluso de innovación social, como algunos estudios recientes sobre Trabajo Social e innovación social muestran (Nandan, London y Bent-Goodley, 2015). Para comprobarlo basta considerar las nuevas políticas de la Unión Europea (Union Innovation 2020), donde se fomentan diversas modalidades de innovación, incluida la innovación social, y no sólo la innovación basada en Investigación + desarrollo (I+D), como en las décadas anteriores $^{1}$. Al revisar la proliferación de artículos y libros es muy común encontrar parejas entre «innovación» y diversas disciplinas. Puede que no 
siempre sean correctos tales emparejamientos y que en la motivación para ello exista cierto oportunismo, dado el auge de los estudios de innovación. Pero en este artículo se propone la hipótesis de que el Trabajo Social ha sido y continúa siendo una actividad basada en la innovación social. Desde su fundación como disciplina hasta la actualidad ha tratado de innovar en muchos sentidos: como práctica, como procedimiento, como agente de cambio social, como reformador, como intermediario en las aplicaciones de las leyes para la protección de los derechos sociales... Y sin embargo, como se recogerá más adelante en este artículo, no ha sido tenido en cuenta como un campo muy propicio para la innovación, debido a la preeminencia del modelo lineal (Investigación+Desarrollo+información $(\mathrm{I}+\mathrm{D}+\mathrm{i})$, en particular en España. El modelo lineal entiende que la investigación científica y los desarrollos tecnológicos (I+D), junto con su ulterior implementación por parte de las empresas y su difusión en los mercados (i), son las únicas fuentes de la innovación. Hoy en día, el paradigma está cambiando y los policy makers europeos han intentado definir y promover el concepto de «innovación social» (BEPA, 2010). Los conceptos adquieren carta de naturaleza social cuando algunos estados los incorporan a sus programas y políticas. Es lo que ha ocurrido con la innovación social. Una vez delineadas sus características, ahora es posible dar el paso de investigar cómo se relaciona este concepto con el Trabajo Social.

Desde ahí que sea importante aportar nuevas líneas argumentales. En primer lugar, conviene entender que existe una cierta autopercepción del Trabajo Social como algo ajeno a la tecnología, entendiendo que es ésta el motor de la innovación. Y es posible que no sea del todo correcto. Existen datos que permiten pensar de otra manera. En segundo lugar, la historia de cómo se constituyen los estudios de Trabajo Social indica que también ese proceso puede leerse a la luz de este concepto ampliado de innovación. En tercer lugar, se expondrá un caso de cómo ha sido posible producir casos de innovación social de una manera muy concreta. Como conclusión se propone que en su conjunto el Trabajo Social es un ámbito de fuerte contenido innovador.

\section{La ampliación del campo en los estudios de innovación}

La innovación — cuál sea su naturaleza, qué valores presenta y cómo pueda favorecerse en los contextos sociales - ha sido una cuestión estudiada desde el primer tercio del siglo pasado, a partir de los trabajos pioneros de Schumpeter (1934, 1939 y 1943). A principios del siglo XXI, los estudios de innovación han terminado por convertirse en un tema imprescindible no solo para la economía y la sociología académicas, sino también para las políticas sociales y económicas de países y entidades supranacionales como la Unión Europea².

En realidad, el desarrollo de los estudios de innovación fue más tardío. Habría que retrotraerse a los años setenta del siglo pasado para comprobar cómo surgieron esos estudios y cómo se fueron extendiendo a numerosas disciplinas de las ciencias sociales. Más recientemente, algunas áreas de humanidades y los propios politólogos han comenzado a desarrollarlos en profundidad. En este casi un siglo de definición del campo, naturalmente, el concepto de innovación ha experimentado diversas transformaciones. En síntesis ha existido un cambio de paradigma de un modelo linear y sistémico — de I+D, donde la ciencia es el motor necesario del cambio- y economicista - las empresas y solo las empresas son las que lideran el campo innovador- hacia un concepto sistémico y ampliado de innovación, en el que diversos agentes sociales pueden ser innovadores y no sólo las empresas.

¿Se puede medir la innovación? y ¿cómo medirla? Éste es el campo de batalla donde se articulan los dos paradigmas. Por una parte se encuentra la visión tradicional -el modelo apoyado en los estudios de Schumpeter - frente a otros modelos alternativos que tratan de ampliar el campo (innovación abierta, innovación de usuarios, etc.). Si se sigue el modelo schumpeteriano, la metodología para medir la innova-

${ }^{1}$ Este artículo basa parte de sus ideas en el libro, Innovations and Values, A European Perspective, de Javier Echeverría, publicado en 2014 por el Center for Basque Studies, University of Nevada, Reno. En él se analiza más ampliamente el giro europeo hacia la innovación social.

2 Para una historia de la innovación es imprescindible el trabajo de Benoît Godin (2015), así como varios artículos previos suyos. 
ción es relativamente sencilla, al estar basada en los indicadores de I+D (Manual de Frascati de la OCDE): hay parámetros susceptibles de medirse, tales como la inversión en $\mathrm{I}+\mathrm{D}$ o las patentes obtenidas. Si bien es cierto que la difusión social no es imprescindible para que haya innovaciones empresariales, como mostró Rogers y se afirma en el Manual de Oslo de la OCDE, la sociedad como tal no es considerada como un factor innovador. Se produce en ella porque ahí se encuentra el espacio para su comercialización, competitividad y difusión, pero no por ella, porque no la produce. Son las empresas las que la crean. Partiendo de ese postulado, las métricas basadas en la I+D permiten clasificar a los diversos países, regiones y empresas, usando los sistemas de indicadores de la OCDE o aquellos de la Unión Europea (EIS, REIS, UIS en la actualidad). Justamente, apoyándose en estas mediciones, fue como el informe Aho (2006) llegó a la conclusión de que la Unión Europea no llegaría a ser líder mundial en la sociedad del conocimiento (Agenda de Lisboa), debido a que otros países eran más innovadores que los europeos.

A principios del siglo XXI han empezado a surgir modelos alternativos al lineal, como el de Von Hippel (2005), de innovación de usuarios, o el de Chesbrough (2006), de innovación abierta. En el modelo de Von Hippel se considera a los usuarios expertos -leading users - como agentes de innovación porque ofrecen conocimiento a las empresas y permiten refinar o transformar sus productos. De esta manera, se comenzó a aceptar que las empresas por si solas no son capaces de producir todo el conocimiento necesario para la innovación. Un ejemplo típico de innovación de usuarios, del que se ocupó a fondo von Hippel, es el de las comunidades de software libre.

\section{La irrupción de la innovación social}

La noción de innovación social apareció entre finales del siglo XX y principios del XXI en cuatro países: Nueva Zelanda, Australia, Canadá y Reino Unido. Los dos últimos son los que gubernamentalmente han impulsado más este nuevo paradigma. Por ejemplo, Canadá en 1997 incluyó las ciencias sociales y humanas como potenciales contribuyentes a los procesos de innovación. El resultado fueron dos informes de expresivo título: Recherche en sciences humaines et sociales et innovations sociales: Contribution à une politique de l'immatériel (1999) e Innovation sociale et innovation technologique: L'apport de la recherche en sciences sociales et humaines. En ellos aparece la expresión «prácticas sociales innovadoras» y directamente «innovación social». Es cierto que en el mundo académico existían diversos estudios y libros con estos conceptos o similares - tal y como muestra Julie Cloutier en Qu'est-ce que la innovation sociale (2003)pero lo importante fue que por primera vez esos conceptos eran básicos para diseñar políticas concretas de innovación en un país ${ }^{3}$. Cloutier definió las innovaciones sociales como aquellas que versan «sobre el consumo, el empleo del tiempo, el entorno familiar, la inserción en el mercado de trabajo, el hábitat, los ingresos, la salud y la seguridad de las personas» (p. 5). Al mismo tiempo era consciente de que este tipo de innovación «se encuentra en la unión de las políticas públicas y de los movimientos sociales: servicios colectivos, prácticas de resistencia, luchas populares, nuevas maneras de producir y consumir» (p. 4). Y también señaló las dificultades para definir el término de forma precisa. Se nota en este informe el énfasis en los movimientos políticos, tanto populares como estatales, y no está tan clara la diferenciación entre innovación social y empresarial, porque de hecho varios apartados del informe se dedican a esta última.

Posteriormente en el Informe Goldenberg (2004) se refinó aún mejor el concepto. Se mantuvo que la innovación social parte, sobre todo, del sector no comercial de la sociedad. Ello implica claramente que no son solo las compañías las que innovan en base a objetivos económicos, sino que también las organizaciones no gubernamentales (ONG), las asociaciones sin ánimo de lucro y el tejido social en general son capaces de innovar, y al margen de los negocios. Ciertamente las fronteras entre innovación empresarial y social son fluidas. En la categorización de Gol-

\footnotetext{
${ }^{3}$ Cloutier señala el inicio en un artículo de James B. Taylor, Introducing Social Innovation (1970). Uno de los estudios más fascinantes sobre cómo se innova pertenece, sin duda, a Michael de Certeau quien, en 1974, describía las «prácticas cotidianas» como innovación en enjambre, de grupos de usuarios. Para el autor las compañías tienen «estrategias» de innovación que el usuario las contrarresta con «tácticas».
} 
denberg se añade, por ejemplo, la noción de «capital social»y «emprendimiento social» para buscar un paralelismo con el mundo empresarial y favorecer una fluidez entre el sector sin ánimo de lucro y el económico. Ciertamente las empresas colaboran con el tercer sector de varias maneras, aunque es obvio que incluso la «responsabilidad social corporativa» — su elemento más cercano a lo social - tiene, en última instancia, un interés de beneficio por medio de la publicidad o el estímulo al consumo.

Siguiendo la estela canadiense, en el Reino Unido la Young Foundation irrumpió en el debate de la innovación social de forma decisiva. Fundada en 1954 por Michael Young, su objetivo era luchar contra la desigualdad y, precisamente, la innovación social fue la herramienta que sintetizó su trayectoria durante casi medio siglo ${ }^{4}$. Geoff Mulgan en colaboración con Simon Tucker, Rushanara Alo y Ben Sanders publicaron, en 2007 dentro de esta Fundación, el libro que se ha convertido en clásico de este tipo de estudios, titulado Social Innovation: What it is, Why it Matters and How Can be Accelerated. En once capítulos y dos anexos el estudio trató de ofrecer una visión exhaustiva de la innovación social por medio de definiciones, comparaciones con los modelos lineales de innovación, distinguiendo entre la innovación en los negocios y la puramente social, introduciendo y calificando como innovaciones sociales movimientos como el feminismo o de gays y lesbianas, identificando agentes tanto en las asociaciones sin ánimo de lucro como en las políticas concretas de países y organizaciones supranacionales, etc. En una breve síntesis inicial, Mulgan (2007) definió así la innovación: «actividades innovadoras y de servicios motivadas por el propósito de dar respuesta a una necesidad social y que son desarrolladas y diseminadas predominantemente por organizaciones cuyos propósitos básicos son sociales» (p. 8). En ese documento se mantuvo una discusión con otros modelos de innovación y se aceptó en parte la validez de algunos de sus enunciados. Por ejemplo, Mulgan se mostró de acuerdo con Schumpeter en que, si no existe difusión social de las invenciones, no se puede hablar en sentido estricto de innovación. La aparición de una innovación social también sirve para abrir un campo para futuras innovaciones; es decir, se producen prototipos, sinergias, replicaciones que se extienden a lo largo del tejido social. Además hay una conexión directa entre la innovación social y económica. Según las cifras que aportó Mulgan, entre el 50 y el 80 por ciento de la actividad económica depende de la innovación en negocios e industria. Pero la innovación social es paralela en importancia a ésta. En este punto, Mulgan retomó estudios previos, como los de von Hippel, Chesbrough y otros autores, para señalar que los usuarios - por ejemplo de Internet, de telefonía, de coches y de otros sistemas tecnológicos - han sido cruciales para redefinir dichos sistemas y aumentar su extensión. Asimismo en el informe de Mulgan se subrayó la importancia de sectores, como el educativo, sanitario y cuidados, estimando que conjuntamente representarían el 30 por ciento del futuro Producto Interior Bruto de los países (p. 5). En este apartado el Trabajo Social obviamente representa un sector crucial para la innovación y Mulgan lo cita expresamente porque considera que hay tres tipos de agentes en la innovación social: individuos, movimientos sociales y organizaciones. Otros agentes, como los gubernamentales y las empresas, pueden coordinarse con proyectos de innovación social, pero la innovación surge de la propia sociedad. Los grupos sociales, en su metáfora, representarían las abejas que sirven para fecundar a los árboles; esto es, empresas e instituciones gubernamentales.

Así pues, desde el punto de vista académico, el campo se creó aproximadamente a principios del presente siglo y aparecieron corrientes diferentes. A la Young Foundation y los trabajos de Chesbrough y Mulgan se añadieron otros, como los de NESTA ${ }^{5}$. Esta última fundación, líder en los estudios de innovación en Europa, acuñó una

${ }^{4}$ En la página web de la organización (www.youngfoundation.org) se lee: we harness the power of social innovation to tackle the root causes of inequality (canalizamos el poder de la innovación social para desterrar las raíces de la desigualdad).

5 Radicado también en el Reino Unido, sus informes han sido extraordinariamente influyentes para delinear las políticas de innovación social en el Reino Unido y parte de la Comunidad Europea (http://www.nesta. org.uk/). Su lema es «somos una organización filantrópica de innovación con la misión de ayudar a las personas y organizaciones a alumbrar grandes ideas» (we are an innovation charity to help people and organizations to bring great ideas to life). 
categoría de gran interés: «la innovación oculta». Con ella trató de interpretar un hecho estadístico importante. En el Reino Unido, solo el 6 por ciento de la innovación existente procede de laboratorios o de investigaciones de carácter científico. Por ello su definición de innovación oculta fue la siguiente:

Actividades de innovación que no se reflejan en los indicadores tradicionales tales como inversiones en investigación y desarrollo formales o en las patentes logradas. Aunque no se mida, con frecuencia la innovación oculta representa la innovación que realmente importa - la innovación que contribuye más directamente a la práctica y comportamiento reales de un sector (NESTA, 2006, p. 4).

Los diversos modelos presentados de innovación social realmente han servido para la creación de políticas específicas: el de Goldeberg influyó en Canadá, Europa y Estados Unidos, favoreciendo en este último caso la creación de la Office of Social Innovation and Civic Participation de la administración Obama en 2009. Así pues, tanto en la academia como en las instituciones gubernamentales se asumió claramente la existencia de procesos de innovación social basados en las actividades de diversos agentes, entre los que se encuentran, o al menos podrían participar, los trabajadores sociales.

\section{Excurso sobre el Trabajo Social y la inno- vación tecnológica de las tecnologías de la in- formación y la comunicación (TIC)}

En un medio innovador como el actual es interesante contemplar cómo se ha relacionado una disciplina, como Trabajo Social, con la tecnología. Esta se ha ido acumulando durante las últimas décadas, y es clarificador cómo se han asimilado las innovaciones técnicas, y si ello revela algo de la propia capacidad innovadora de los trabajadores sociales. Siguiendo a Alonso (2015, pp. 35 y ss.) se ha establecido casi como idea asumida, la resistencia de los trabajadores sociales tanto a la innovación como a la introducción de herramientas tecnológicas en su uso profesional. En cierto sentido esta impresión, no del todo justificada, puede entenderse con las situaciones de otras profesiones y disciplinas. El cambio suscitado por las TIC ha supuesto una transformación profunda que está siendo vivida como disruptiva en muchas ocasiones. Los sucesivos cambios tecnológicos han ocurrido a una velocidad demasiado grande como para poder asimilarlos por completo, y no solo en la práctica del Trabajo Social. Históricamente la introducción de ordenadores en áreas distintas a la de informática se realizó de forma abrupta. Muchas veces no se tuvo en cuenta a los usuarios de las herramientas y en numerosas ocasiones no existió una preparación previa de los futuros usuarios. El gran avance en usabilidad, que se ha producido en los últimos años, indica también que el diseño de las herramientas ha tenido que ser repensado para tomar en consideración a quienes no eran técnicos, pero que debían utilizar los ordenadores. Un breve recorrido por los inicios de la informatización de áreas no científicas muestra numerosos casos de esta índole.

A pesar de ello, los primeros escritos sobre el uso de tecnología por los trabajadores sociales son de 1985, cuando se publica la revista Computing and Social Services. Ésta cambiará su nombre por más tarde Technology and Human Services hasta el presente, lo que indica la necesidad de explorar, compartir y repensar la tecnología desde el punto de vista de los trabajadores sociales. Asimismo en la práctica profesional se comienza a experimentar, en la década de 1990 , sobre las posibilidades de la ayuda on-line. En este momento confluyen otras disciplinas con profesionales en relación con pacientes o usuarios, como psicólogos, psiquiatras y terapeutas, que encuentran en los ordenadores en red una posibilidad que requiere ciertas cautelas. Las cuestiones éticas y deontológicas también han sido atendidas inmediatamente, como muestra el temprano interés por saber qué ocurre con el almacenamiento de los datos ya en los años 1970 (Parker, Swope y Baker, 1990). La introducción de estas tecnologías coloca en el foco de atención problemas tales como la relación personal con los usuarios (Reamer, 2013), su privacidad y confidencialidad (Kolmes \& Taube, 2010) o la brecha digital (Parker-Oliver y Demiris, 2006). La propia National Asociation for Social Workers (NASW) lanzó en 2005 un informe para los estándares éticos y deontológicos que había que aplicar a la tecnología, cuando es herramienta de los trabajadores sociales.

\section{La innovación y el Trabajo Social}

Con la perspectiva delineada en la primera parte del artículo se puede aceptar que el Trabajo So- 
cial es compatible con una teoría ampliada de la innovación. Si se abandonan las preconcepciones respecto a productos, marketing, empresas y logística está claro que la evolución de esta profesión es indudablemente un desarrollo innovador. La propia historia lo indica. La caridad ha sido un valor más o menos promocionado en las sociedades europeas desde antiguo, bien como resultado de valores religiosos o bien de valores humanistas. Sin embargo la irrupción de la Revolución Industrial y el consiguiente flujo migratorio a las ciudades supusieron un grave problema, expresado en situaciones nuevas, como el desempleo, la falta de alojamiento, las enfermedades provocadas por la falta de higiene, la pauperización de familias e individuos y la malnutrición, entre otros. Se trataba entonces de una situación que, en términos de Mulgan, suponía un problema acuciante para la sociedad de la época (pressing issues). El cambio innovador, que se propone precisamente con las Charity Organization Societies, implica reorganizar los recursos, las organizaciones y acciones con la introducción de un enfoque científico de los problemas. Se recolecta información sobre los usuarios de forma centralizada, a fin de optimizar los recursos y lograr una mayor efectividad. La transformación progresiva de los voluntarios y colaboradores en profesionales es también una solución novedosa a la caridad, entendida hasta entonces como un compromiso voluntario y sin beneficio económico. Esta primera organización de las sociedades caritativas, que se instituye en Inglaterra, se exporta rápidamente a otros países, como EE.UU. La replicación indica asimismo la difusión de la innovación hasta que el Trabajo Social, como disciplina, se extiende por todo el mundo. La profesionalización iniciada por Richmond supone una innovación ${ }^{6}$ de gran calado, porque ha cambiado no solo la actividad de los trabajadores sociales sino también las propias leyes que los estados han ido creando sucesivamente hasta llegar al Estado del bienestar. Ahora la caridad es sustituida por los derechos básicos, y no es algo gratuito sino un compromiso de carácter político.

Si la lucha por el sufragio universal, por la equiparación entre géneros, por la tolerancia religiosa o cultural son elementos innovadores dentro del devenir social, también merecen el mismo tratamiento las propuestas activistas de los trabajadores sociales. Es difícil separar unas de otras. Recuérdese que el Trabajo Social se define entre dos ejes: el cambio individual y el cambio social, dependiendo de dónde se sitúe la raíz del problema (Barahona, 2015). Es común la definición de los profesionales del Trabajo Social como agentes de cambio. En cualquier caso se trata de una innovación. Las propuestas en política social provienen de los organismos públicos, pero son los trabajadores sociales quienes las reinterpretan al aplicarlas. Asimismo su experiencia permite extraer conocimiento para corregir los errores que los propios legisladores y partidos políticos deben asumir. Sin la capacidad de innovar en esas políticas posiblemente muchas de ellas fracasarían. Añádase a esto la situación contemporánea en la que el denominado Estado del bienestar se entiende en retroceso. Sin embargo los problemas sociales, la exclusión, el paro, la falta de atención en las enfermedades crónicas y mentales, y la consiguiente marginación de capas sociales cada vez más numerosas están ahí. Por ello parece adecuado aplicar la definición de Mulgan y afirmar que el Trabajo Social contemporáneo trata de dar respuesta a los problemas acuciantes de la sociedad actual. En realidad esta necesidad de innovación puede interpretarse como una obligación para los trabajadores y las trabajadoras sociales (Pawar, 2014).

Una forma de interpretar la innovación en Trabajo Social podría ser entender a los trabajadores sociales como catalizadores del cambio social entre los individuos y las comunidades de afectados y las instituciones o grupos gubernamentales (Nandan, London y Bent-Goodley, 2015). Se habla así de «emprendedores» y de «intra-emprendedores» sociales: los primeros se definirían como quienes tratan de promover políticas de cambio en alianza con comunidades e instituciones y los segundos como aquellos individuos que, trabajando dentro de una corporación o una agencia gubernamental, tratan de innovar dentro de ella. Estas nuevas figuras se deben a la volatilidad presente donde la filantropía, la caridad, los derechos y las políticas sociales cambian a gran velocidad y donde los problemas, como se ha indicado anteriormente, se agravan.

${ }^{6}$ Ivan Illich (1981) sostendrá que este cambio de la caridad supone una innovación disruptiva y contraproducente. 


\section{Caso de estudio}

El caso de innovación en Trabajo Social es un proyecto liderado por María José Barahona, cuyo título es «Unidad móvil para la atención a mujeres que ejercen la prostitución». Este proyecto se diseña como un estudio y análisis de los proyectos outreach existentes en ese momento en Norteamérica (1989) para intentar contener lo que se considera la pandemia del siglo XX; el $\mathrm{VIH} / \mathrm{SIDA}^{7}$. Se entiende que la prostitución es un vector importante de infección entre la población heterosexual y que es necesario atajarlo. $\mathrm{Pa}$ ra ello se fomenta el uso de preservativos en la calle, entre las prostitutas, y se les recompensa a las mismas con algún tipo de premio (preservativos, kits de limpieza y de jeringuillas, comidas gratis en McDonald's, por ejemplo). Otros países se suman a la necesidad de organizar algún tipo de campaña, como en Francia, Italia, Portugal, Holanda y Alemania.

El proyecto se presenta con un carácter innovador por la insatisfacción del modelo previo, que no puede ser directamente adaptado. Es necesario reformar, adaptar y orientar el tipo de intervención al contexto y la realidad en donde se realiza. Primeramente acercarse a las prostitutas como vector de infección significa, de alguna manera, culpabilizarlas de la extensión de la epidemia. En este sentido se vuelve de forma indirecta al reglamentismo practicado en el siglo XIX, cuando a las prostitutas se les somete al control estatal de su salud genital. No se tiene en cuenta que son los clientes - algunos de ellos dispuestos a pagar más por no usar los preservativos - el otro vector de infección. Añádase a ello que la situación de las prostitutas es el resultado de condiciones adversas, tanto económicas como delictivas (trata), que son las que les fuerzan a ejercer. Por lo tanto, hay que entender que son esencialmente víctimas y no culpables de extender la enfermedad. Por ello el lema de la intervención es «cuídate a ti para ti». No se trata de asegurar un sexo sin riesgo para los clientes, sino la percepción de salud de las propias prostitutas, quienes son las verdaderas víctimas de las enfermedades de transmisión sexual, porque no pueden elegir.
Hay que tener en cuenta las condiciones cambiantes de la prostitución a lo largo del tiempo. Si bien formalmente la de prostituta es una categoría, las prostitutas concretas difieren en muchos aspectos. Por ejemplo, los años 1980 muestran en nuestro país un tipo de prostituta nacional y con cierta frecuencia con adicciones a distintas sustancias. Con el tiempo han aparecido cambios en la tipología, debido fundamentalmente a su procedencia - subsaharianas, sudamericanas, de países del Este, etc. - con sus consiguientes diferencias culturales. El proyecto tomó muy en serio esta diversidad y la necesidad de adaptarse a una realidad social cambiante. La intervención se plantea por ello con dos principios complementarios. El primero es la constancia y rigurosidad de horarios para la intervención, combinada con la flexibilidad que cada caso particular requiere. $\mathrm{Pa}$ ra ello el espacio de intervención, una autocaravana, ha de asegurar cierta intimidad. No se trata solo de repartir kits o preservativos, sino de llegar a la persona concreta, crear cierta intimidad entre el trabajador o trabajadora social y la usuaria, y trabajar a partir de la confianza.

Repartir solo jeringuillas o preservativos, como en los proyectos precedentes, es insatisfactorio porque no tiene en cuenta los cambios en la prostitución y las situaciones individuales. De hecho si primeramente se trata de un apoyo sanitario y social, luego se añade también el legal. La inmigración es un problema creciente y la trata es un delito. Si en los años 1980 la cuestión era de salud y de encontrar alternativas para las prostitutas, a partir de los años 1990 el tráfico de mujeres y la inmigración alegal son problemas añadidos que requieren respuestas de otros contextos, como del derecho. De ahí precisamente la necesidad de la flexibilidad. Se trata de proporcionar, entonces, una intervención holística del problema y que, en cierta medida, supone recuperar la tradición del Trabajo Social de ir al usuario - intervención en medio abierto - en vez de esperar que éste vaya a la institución y aguarde a ser atendido - intervención en el despacho. Como resultado esta práctica se ha estabilizado en España y hay diversas ONG que continúan con diferentes proyectos.

${ }^{7}$ Se trata del Project Street Beat, un programa perteneciente a la Ong Planned Parenthood de Nueva York (https://www.plannedparenthood.org/planned-parenthood-new-york-city) que en 1989 organizó visitas al Bronx en una furgoneta. Con ella proporcionaban preservativos, kits de limpieza y cupones para comer en McDonalds. (http://www.nytimes.com/1989/10/06/nyregion/van-is-hope-for-bronx-throwaways.html). 
En resumidas cuentas, este proyecto se presenta como una innovación respecto a otros más anticuados. Supone reorganizar el prototipo de innovación y confrontar con flexibilidad un ámbito cambiante, con demandas diferentes según el momento, pero al tiempo de extrema urgencia. El modelo puede ser replicado en otros países, sociedades o culturas, y de hecho así ha ocurrido. En cada caso es necesario adaptarlo a las situaciones concretas, refinar sus características y ampliar sus objetivos. Supone al tiempo un cambio de una forma de entender la intervención - burocratizada y de despacho en un momento determinado - y volver a los orígenes primeros del Trabajo Social. En este sentido es una auténtica innovación.

\section{Conclusiones}

Como se ha analizado, el Trabajo Social mantiene una estrecha relación con la innovación social. No es casual que dos organizaciones estrechamente ligadas al filantropismo y la caridad, como la Young Foundation y la NESTA sean agentes claves para definir este concepto. Por lo tanto, si se amplía el campo de investigación y se cambia de paradigma, entonces es posible encontrar la congruencia para la innovación dentro de esta disciplina. Por ello esta ampliación encuentra en las características esenciales del propio Trabajo Social el elemento innovador, porque en esa dualidad entre activismo y agente de las políticas sociales se encuentra ese potencial. Por otra parte, la sensación — cada vez más recurrente entre los trabajadores y las trabajadoras sociales - es que, ante un Estado del bienestar en desaparición y la retirada de las posiciones gubernamentales, es necesario innovar en la práctica para poder enfrentarse a los retos presentes y futuros. Al tiempo, se necesita una actitud más flexible ante los cambios sociales que muchas veces no pueden atenderse en unas instituciones burocratizadas y rígidas. Se puede decir, entonces, que existe cada vez más la convicción profesional de que o se innova en Trabajo Social o éste perderá el avance que ha logrado en este tiempo.

\section{Referencias bibliográficas}

Aho, E., Cornu, J., Georghiou, L. y Subira, A. (2006). Creating an innovative Europe. Report of the Independent Expert Group on R\&D and Innovation appointed following the Hampton Court Summit. Luxembourgo: Comisión Europea.

Alonso, D. (2015). Trabajo social y tecnología: Aceptación y uso entre profesionales en formación. [Tesis doctoral]Ma,drid: Facultad de Trabajo Social: Universidad Complutense, 20 de noviembre.

Barahona, M.J. (2015). Prostitución, abolicionismo y Trabajo Social. Las actitudes de los y las profesionales en la Comunidad de Madrid. [Tesis doctoral] Madrid: Facultad de Trabajo Social: Universidad Complutense.

BEPA. (2010). Empowering people, driving change: Social innovation in the European Union. Report prepared by A. Hubert, M. Bonifacio and J. Konings, Bureau of European Policy Advisers. Recuperado de: http://ec.europa.eu/bepa/pdf/publications_pdf/social_innovation.pdf.

Chesbrough, H.W. (2006). Open innovation: The new imperative for creating and profiting from technology. Harvard Business Press.

Cloutier, J. (2003). Qu'est-ce que l'innovation sociale? Les cahiers du Crises. Collection Études theóriques, ET0314. Québec: Crises.

Godin, B. (2008). Innovation: the History of a Category. Montreal: INRS, Project of Intellectual History of Innovation.

Godin, B. (2010). Social Innovation. Montreal: INRS, Project of Intellectual History of Innovation.

Godin, B. (2015). Innovation Contested - The Idea of Innovation Over the Centuries. Londres: Routledge.

Goldenberg, M. (2004). Social Innovation in Canada. How the non-profit sector serves Canadians... and how it can serve them better. Ottawa: Canada Policy Research Networks.

Gouvernement de Québec, Conseil québécois de la recherche sociale. (CQRS) (1999). En: Camil Bouchard y Groupe de travail sur l'innovation sociale (GTIS), Recherche en sciences humaines et sociales et innovations sociales. Contribution á une politique de l'immatériel. Québec: Les Publications du Québec.

Illich, I. (1981). Profesiones inhabilitantes. Barcelona: Blume. 
Kolmes, K. y Taube, D.O. (2010). Clinical implications of therapist-client interactions on the Internet: Boundary considerations in cyberspace. San Francisco: American Psychological Association Division of Psychotherapy.

Mulgan, G., Tucker S., Rushanara, A. y Sanders, B. (2007). Social innovation: what it is, why it matters and how it can be accelerated. Oxford: Oxford Said Business School.

Nandan, M., London, M. y Bent-Goodley, T. (2015). Social Workers as Social Change Agents: Social Innovation, Social Intrapreneurship, and Social Entrepreneurship. Human Service Organizations: Management, Leadership \& Governance, 39(1), 38-56.

NASW. (2005). Standards for Technology and Social Work Practice. Recuperado de: http://www.socialworkers.org/practice/standards/NASWTechnologyStandards.pdf (consultado el 23-01-2016).

NESTA. (2006). The Innovation Gap: Why Policy Needs to Reflect the Reality of Innovation in the UK.Policy Briefing IG/02, NESTA Policy and Research Unit. London: NESTA.

OECD. (2002). Frascati Manual, Proposed Standard Practice for Surveys on Research and Experimental Development. París: OECD.

OECD. (2005 [1992]). OECD Proposed Guidelines for Collecting and Interpreting Technological Innovation Data: Oslo Manual. París: OECD.

Parker-Oliver, D. y Demiris, G. (2006). Social work informatics: A new specialty. Social Work, 51(2), 127-134.

Parker, D.B., Swope, S. y Baker, B.N. (1990). Ethical conflicts: in information and computer science, technology, and business. Massachusetts: QED Information Sciences, Inc.

Pawar, M. (2014). Social Challenges and Policy Innovations by Social Workers in Australia. Center for Social Development, Saint Luis: Washington University Saint Luis. Recuperado de: http://csd.wustl.edu/Publications/Documents/WP14-10.pdf. (Consultado el 22/01/2016).

Reamer, F.G. (2013). The digital and electronic revolution in social work: Rethinking the meaning of ethical practice. Ethics and social welfare, 7(1), 2-19.

Rogers, E.K. (1995). Diffusion of Innovations. (4' edición). Nueva York: The Free Press.

Schumpeter, J.A. (1934). The Theory of Economic Development: An Inquiry into Profits, Capital, Credit, Interest, and the Business Cycle. Harvard: Harvard University Press.

Schumpeter, J.A. (2008[1939]). Business Cycles. Nueva York, Toronto, Londres: McGraw-Hill. (Edición electrónica de D. Lagrange). Quebec, Canadá, 2008, 80 (consultado el 15-8-2011).

Schumpeter, J. A. (1975[1942]). Capitalism, Socialism and Democracy. (2 ${ }^{\text {a }}$ edición). Nueva York: Harper \& Row.

Von Hippel, E. (2006). Democratizing Innovation. MIT Press Books, 1. 
